\title{
Advantages of Micro-XRF in Forensic Science: From Large Areas to Minute Amounts
}

\author{
Max Bügler ${ }^{1}$, Falk Reinhardt ${ }^{1}$, Samuel Scheller ${ }^{1}$, Roald Tagle ${ }^{1}$, and Timo Wolff ${ }^{1}$ \\ 1. Bruker Nano GmbH, Am Studio 2D, Berlin, Germany
}

Micro-XRF, as an analytical tool, combines several features, making it ideal for investigation of forensic samples. The technique can be used to scan large areas, provides simultaneous information on multiple elements down to the trace element level, can be applied under ambient conditions and is non-invasive. Furthermore, the requirement in forensics to not alter or damage the specimen suits this technique perfectly, as it requires no or little preparation of the sample, other than fixing it to the stage or flattening the specimen, as can be seen in Figure 1a.

This all-round capability and non-invasive nature, has led numerous forensic institutes and departments to use this technique in many investigations. Some use it as the first investigative technique, as it can provide many insights and discoveries about the specimen without any preparation or even vacuum. Although vacuum can be applied and adjusted from 1 mbar to atmospheric pressure, which will improve light element detection and sensitivity, it is not necessary for heavier elements, from calcium and above. If light element detection at atmospheric pressure were required, helium purging remains a viable option.

Over the last 20 years Micro-XRF has been used extensively in forensic shooting distance determination (see Figure 1b). For this forensic evidence, a shot-through sample is scanned and the gun shot residue pattern is compared to that of a series of reference shootings. This scientific approach requires several of the analytical strengths of micro-XRF: Large areas must be assessed in a spatially resolved manner. The amount of sample material diminishes with larger distances to a point were trace element sensitivity is required. Above all, the sample must remain undamaged or free of manipulation as it might be needed for further investigations or as evidence at court. Again, the non-intrusive manner of the technique, requiring little or no preparation makes it ideal for this application.

The forensic community started using this technique for other applications, such as determining differences in or origins of glass, glass fragments or even sands from crime scenes. This technique allows for simultaneous detection of all elements from $\mathrm{Na}$ to $\mathrm{U}$ down to the trace level. The doping agents that give glass its properties, such as color or strength, are generally all in the parts per million (ppm) or even smaller (Figure 2), but can be used to uniquely identify and correlate the origin of such specimens. This is made use of in test methods such as the ASTM2926 [1]. Here again the micro-XRF technique is ideal, as it can analyze even small splinters or fragments.

In this paper, the authors want to present a brief introduction to the fundamentals of the technique, some of the instruments used in forensics as well as their specifications. From there, the conditions and timeframes for each of the numerous examples of measurements performed with micro-XRF for forensic investigations will be given. For each example, the supporting evidence will be shown and summarized. 


\section{References:}

[1] ASTM E2926-17, Standard Test Method for Forensic Comparison of Glass Using Micro X-ray Fluorescence ( $\mu$-XRF) Spectrometry, ASTM International, West Conshohocken, PA (2017) www.astm.org
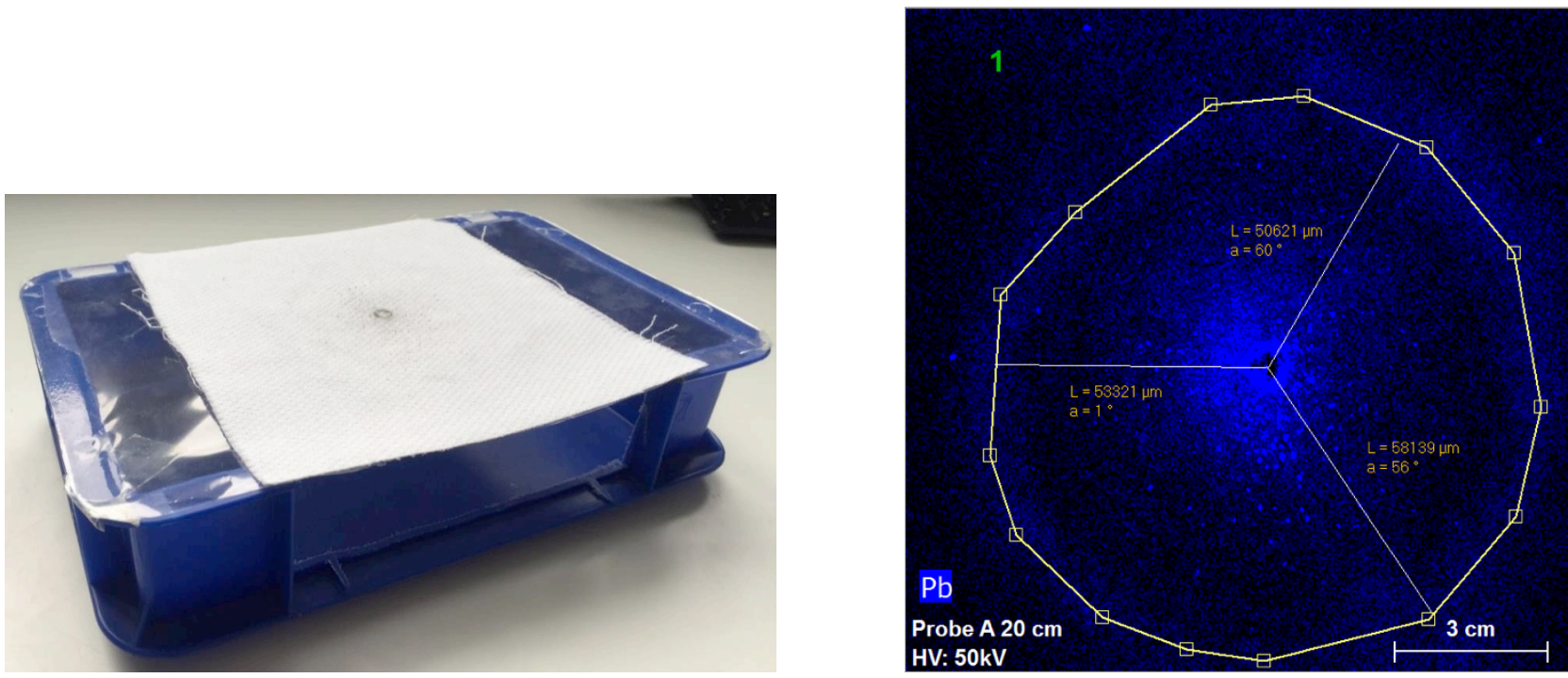

Figure 1. a) Shot-through cloth sample on container with foil to reduce background scatter. b) $\mathrm{Pb}$ distribution of a gunshot residue shot at $20 \mathrm{~cm}$ distance. Quantification of geometric properties of the pattern.

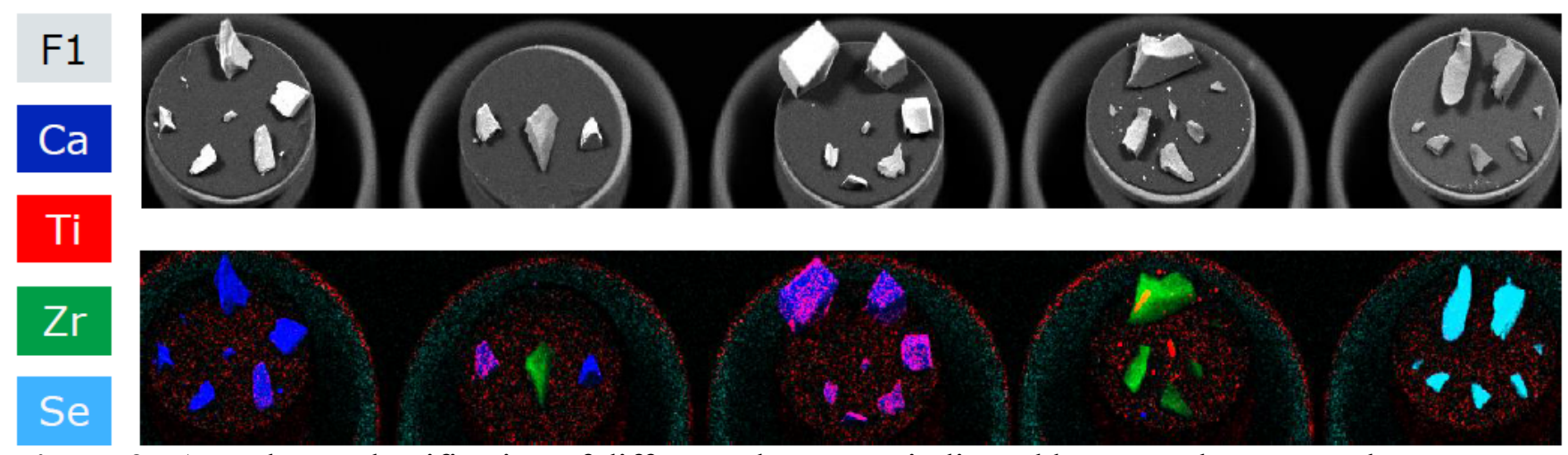

Figure 2. At a glance classification of different glass types indicated by trace element markers 